\title{
Avaliação das potencialidades dos extratos vegetais de jurema preta (Mimosa tenuiflora) e cajueiro (Anacardium occidentale L.) para uso em embalagens ativas antimicrobianas e antioxidantes
}

\section{Evaluation of the potentials of jurema preta (Mimosa tenuiflora) and cajueiro (Anacardium occidentale L.) extracts for use in antimicrobials and antioxidants active packaging}

Ivo Diego de Lima Silva ${ }^{1}$, Flávia Suellen Melo de Oliveira ${ }^{2}$, Michelle Felix de Andrade ${ }^{2}$, Andréa Monteiro Santana Silva Brito ${ }^{3}$, Fernando Hallwass ${ }^{4}$, Glória Maria Vinhas ${ }^{2}$

\footnotetext{
${ }^{1}$ Programa de pós-graduação em Ciência de Materiais, Universidade Federal de Pernambuco (PGMtr-UFPE), Av. da Arquitetura S/N, CEP: 50740-540, Recife, Pernambuco, Brasil.

${ }^{2}$ Departamento de Engenharia Química, Universidade Federal de Pernambuco (DEQ/UFPE), Av. dos Economistas S/N, CEP: 50740-590, Recife, Pernambuco, Brasil.

${ }^{3}$ Unidade Acadêmica de Serra Talhada, Universidade Federal Rural de Pernambuco (UAST/UFRPE), Av. Gregório Ferraz Nogueira S/N, CEP: 56909-535, Serra Talhada, Pernambuco, Brasil

${ }^{4}$ Departamento de Química Fundamental, Universidade Federal Rural de Pernambuco (DQF/UFPE), Av. da Arquitetura, 540, CEP: 50740-540, Recife, Pernambuco, Brasil.

e-mail: ivo.diego91@gmail.com, efsm.oliveira@outlook.com; gmvinhas@yahoo.com.br, mifelixsilva@hotmail.com, andrea.monteiros@ufrpe.br, hallwass@ufpe.br
}

\section{RESUMO}

As embalagens têm como função básica proteger o alimento e garantir a sua integridade comercial. Podem ser produzidas a partir de materiais biodegradáveis com funcionalidades específicas, tais como ação antimicrobiana e antioxidante. Além disso, pesquisas vêm sendo desenvolvidas de modo a substituir os aditivos sintéticos por outros naturais, como os extratos vegetais, devido aos compostos químicos presentes em suas misturas. O poli(butileno adipato co-tereftalato) - (PBAT) vem sendo extensivamente estudado como uma possível substituição aos polímeros convencionais devido à sua biodegradabilidade. Desse modo, o objetivo desse trabalho foi avaliar as potencialidades dos extratos vegetais de jurema preta (Mimosa tenuiflora) e cajueiro (Anacardium occidentale Linn), a fim de aplicá-los como aditivos em embalagens de PBAT com propriedades ativas antioxidantes e antimicrobianas. Os extratos foram obtidos pela técnica de extração Sohxlet em três diferentes tempos, 4, 6 e 8 horas, cujo melhor rendimento ocorreu nos tempos de 6 horas para jurema preta e 4 horas para cajueiro. No teste de presença de flavonóides o extrato de jurema preta apresentou maior concentração e no teste de fenólicos totais, utilizando o método de Folin-Ciocalteau, o cajueiro obteve maior concentração. Ambos os extratos apresentaram capacidades antioxidantes significativas pelos dois métodos, fosfomolibdênio e DPPH. cajueiro apresentou melhor halo de inibição frente ao Staphylococcus aureus, e, por isso, foi determinada a sua concentração mínima inibitória (CMI), 0,0170 g.mL $\mathrm{m}^{-1}$. Esse extrato foi incorporado ao PBAT, para o ensaio de migração no meio etanólico, por meio do qual foi determinado um modelo cinético e foi observado que a taxa de migração, $0,0167 \mu \mathrm{g} \cdot \mathrm{mL}^{-1} \cdot \mathrm{min}^{-1}$, foi reduzida em cerca de um dia, indicando bom potencial do extrato para esses tipos de embalagem.

Palavras-chave: embalagens ativas; embalagens antioxidantes; embalagens biodegradáveis; extratos vegetais; cinética de migração.

\section{ABSTRACT}


Packaging has the basic function of protecting the food and ensuring its commercial integrity. They can be produced from biodegradable materials with specific functionalities such as antimicrobial and antioxidant action. In addition, research has been carried out to replace synthetic additives with natural additives, such as plant extracts, due to the chemical compounds present in their mixtures. Poly (butylene adipate coterephthalate) - (PBAT) has been extensively studied as a possible replacement for conventional polymers due to its biodegradability. Thus, the objective of this work was to evaluate the potentialities of jurema preta (Mimosa tenuiflora) and cajueiro (Anacardium occidentale Linn) plant extracts in order to apply them as additives in PBAT packages with antioxidant and antimicrobial active properties. The extracts were obtained by the Sohxlet extraction technique at three different times, 4,6 and 8 hours, with the best yield at 6 hours for jurema preta and 4 hours for cajueiro. In the flavonoid presence test the jurema preta extract presented higher concentration and in the total phenolics test, using the Folin-Ciocalteau method, the cashew tree obtained the highest concentration. Both extracts showed significant antioxidant capacities by the two methods, phosphomolybdenum and DPPH. Cashew tree presented better inhibition halo against Staphylococcus aureus, and therefore its minimum inhibitory concentration (MIC) was determined, $0.0170 \mathrm{~g} \cdot \mathrm{mL}^{-1}$. This extract was incorporated into the PBAT for the ethanolic migration test, by means of which a kinetic model was determined and it was observed that the migration rate, $0.0167 \mu \mathrm{g} \cdot \mathrm{mL}^{-1} \cdot \mathrm{min}^{-1}$, was reduced by about a day, indicating good extract potential for these types of packaging.

Keywords: active packaging; antioxidant packaging; biodegradable packaging; plant extracts; kinetics of migration.

\section{INTRODUÇÃO}

As embalagens tradicionais apresentam como função a proteção ao alimento contra ações químicas, físicas ou microbiológicas de deterioração durante toda a sua validade comercial [1], agindo como uma barreira contra o meio externo. Com a finalidade de desenvolver embalagens que não só protejam, mas também interajam com o produto ampliando o seu tempo de prateleira e fornecendo maior segurança alimentar [2], surgiram as embalagens denominadas ativas.

As embalagens ativas são um sistema inovador que favorece a interação entre o produto e os materiais da embalagem, proporcionando um aumento na vida útil do alimento e assegurando a sua qualidade final e sua durabilidade [3]. Nessa nova classe de embalagem, destacam-se as embalagens ativas antimicrobianas e antioxidantes, que são baseadas na liberação de aditivos e/ou conservantes na superfície dos alimentos embalados, a partir da matriz polimérica, que irão proporcionar a inibição do crescimento microbiológico do meio [4] e inibição ou retardamento significativo do processo oxidativo, diminuindo a introdução direta dessas substâncias nos alimentos, uma vez que a difusão delas ocorrerá de forma controlada, ficando retidas, principalmente, nas superfícies dos produtos.

Vários agentes antimicrobianos e antioxidantes podem ser incorporados nesse tipo de embalagem, produzidas com ou sem migração do composto. Em geral, esses agentes podem ser classificados em sintéticos e naturais [5], sendo os sintéticos, ainda, os mais utilizados.

Como uma possível substituição aos aditivos sintéticos, a exploração da vegetação do sertão nordestino apresenta uma grande diversidade de plantas com potencialidade para extração de componentes ativos para o combate de micro-organismos e, dentre as espécies desse bioma, estão: jurema preta (Mimosa tenuiflora) e cajueiro (Anacardium occidentale Linn), uma vez que, segundo Lozoya et al., [6], a primeira possui atividade antimicrobiana comprovada em Staphylococcus epidermitis, Escherichia coli, Pseudomonas aeruginosa, Micrococcus luteus e Acinetobacter calcoaceticus e, de acordo com Laurens et al., [7], a segunda possui atividade antimicrobiana verificada em Proteus morganii, Pseudomonas aeruginosa, Staphylococcus aureus, Escherichia coli e Salmonella typhi.

Outra característica das embalagens ativas é o tipo de polímero utilizado, logo, aliando as questões ambientais e econômicas [8], o poli (butileno adipato co-tereftalato) (PBAT), vem sendo estudado como uma alternativa para substituição dos polímeros convencionais. O PBAT é um copoliéster alifático-aromático produzido por reação de condensação a partir do 1,4 butanodiol, ácido adípico e ácido tereftálico, apresenta boas propriedades térmicas e mecânicas, sendo similar as características encontradas no polietileno de baixa densidade [9].

O PBAT tem como nome comercial ECOFLEX, sendo produzido pela BASF (Alemanha). As propriedades mecânicas e físicas estão relacionadas com as unidades aromáticas e a biodegradabilidade às unidades alifáticas. O PBAT pode ser aplicado na produção de embalagens de alimentos, sacos plásticos compostáveis para jardinagem e no uso agrícola, além de seu uso em revestimentos resistentes à água [10].

Assim, com o propósito de substituir os conservantes sintéticos nos alimentos por aditivos naturais nas embalagens, este trabalho tem como objetivo geral avaliar as potencialidades dos extratos vegetais de 
jurema preta (Mimosa tenuiflora) e cajueiro (Anacardium occidentale Linn), como aditivos antioxidantes e antimicrobianos em embalagens de PBAT.

\section{MATERIAIS E MÉTODOS}

\subsection{Extrações vegetais}

Para a extração foi utilizado $200 \mathrm{~mL}$ de etanol e, aproximadamente, $20 \mathrm{~g}$ das cascas de jurema preta e cajueiro. As plantas são oriundas do município de Serra Talhada do estado de Pernambuco. O material vegetal foi triturado e inserido em cartuchos confeccionados com papel de filtro. Posteriormente, adicionouse o cartucho recheado na câmara de amostra do extrator soxhlet.

As extrações foram realizadas em triplicatas nos tempos de extração de 4, 6 e 8 horas. Para a análise do rendimento foi inicialmente necessário medir o volume final obtido da extração com o auxílio de uma proveta, em seguida, foi retirado $1 \mathrm{~mL}$ da solução e adicionado em um vidro de relógio previamente pesado, colocado em uma estufa com circulação de ar na temperatura de $70{ }^{\circ} \mathrm{C}$ para a evaporação do etanol. Posteriormente, pesou-se o vidro de relógio até a obtenção de massa constante. Através desses procedimentos e utilizando a equação 1 , foi obtida a massa bruta total do extrato vegetal.

Massa $_{\text {total }}=$ Massa $_{\text {extrato }(1 \mathrm{~mL})}$ XVolume Total

O rendimento percentual do extrato vegetal em relação a matéria-prima do material vegetal triturado foi adquirido através da equação 2 .

Rendimento $(\%)=\frac{\text { Massa total do extrato }(g)}{\text { Massa da matéria-prima }(g)} \times 100$

O extrato vegetal foi concentrado através da rotaevaporação. Para isso, levou-se a solução para um rotaevaporador, na temperatura do banho de $60^{\circ} \mathrm{C}$ e pressão de $550-600 \mathrm{mmHg}$.

\subsection{Teste de presença de flavonoides}

Para a realização do teste de presença de flavonoides foi utilizada a metodologia adaptada de Oliveira $e t$ al., [11]. Primeiramente, foram preparadas as soluções metanólicas dos extratos vegetais secos $\left(1000 \mu \mathrm{g} \cdot \mathrm{mL}^{-}\right.$ ${ }^{1}$ ) e de cloreto de alumínio $5 \%(\mathrm{~m} / \mathrm{v})$. Logo após, em um tubo de ensaio, foram adicionados $2 \mathrm{~mL}$ da solução do extrato vegetal, $1 \mathrm{~mL}$ da solução metanólica de cloreto de alumínio e $2 \mathrm{~mL}$ de metanol PA. Para o branco, foram utilizados $2 \mathrm{~mL}$ de metanol PA no lugar da solução do extrato vegetal. Aguardou-se um período de 30 minutos na ausência de luz, em seguida foram retiradas as leituras em um espectrofotômetro UV/VIS no comprimento de onda de $420 \mathrm{~nm}$. Todas as amostras foram realizadas em quintuplicatas. A curva de calibração foi obtida através do padrão de quercetina PA.

\subsection{Determinação dos fenólicos totais}

Para a determinação dos fenólicos totais nos extratos vegetais, foi utilizado o método espectrofotométrico de Folin-Ciocalteau [12]. Inicialmente foi preparado uma solução etanólica do extrato vegetal $\left(200 \mu \mathrm{g} \cdot \mathrm{mL}^{-1}\right)$. Em seguida, foi adicionado em um tubo de ensaio $8 \mathrm{~mL}$ de água destilada, 0,5 $\mathrm{mL}$ do reagente de FolinCiocalteau e 0,5 mL da solução do extrato vegetal e posto a agitar por 3 minutos e, após esse tempo, adicionou-se $1 \mathrm{~mL}$ de uma solução de carbonato de sódio saturada. A solução foi deixada em repouso por um período de 60 minutos e, em seguida, medida as absorbâncias no comprimento de onda de $720 \mathrm{~nm}$. O experimento foi feito na ausência de luz e em triplicata. Para o branco, utilizou-se etanol PA e soluções etanólicas de ácido gálico como padrão de referência para construir uma curva de calibração.

\subsection{Capacidade antioxidante total dos extratos vegetais}

A capacidade antioxidante total dos extratos vegetais de jurema preta e cajueiro foram determinadas a partir do método de redução do fosfomolibdênio [13]. Para isso, foram misturados, em tubos de ensaio, alíquotas de $0,3 \mathrm{~mL}$ do extrato, na concentração de $200 \mu \mathrm{g} / \mathrm{mL}$ em metanol, com $3 \mathrm{~mL}$ de uma solução reagente composta de ácido sulfúrico $\left(0,6\right.$ mol. $\left.\mathrm{L}^{-1}\right)$, fosfato de sódio $\left(0,028 \mathrm{~mol} . \mathrm{L}^{-1}\right)$ e molibdato de amônio $\left(0,004 \mathrm{~mol} . \mathrm{L}^{-1}\right)$. Como branco, utilizou-se $0,3 \mathrm{~mL}$ de metanol ao invés da solução do extrato e, para o padrão, ácido ascórbico. Os tubos de ensaio foram fechados e mantidos no banho maria a $95{ }^{\circ} \mathrm{C}$ por $90 \mathrm{~min}$. Após o resfriamento, a leitura das absorbâncias foi feita em espectrofotômetro UV/Vis no comprimento de onda de $695 \mathrm{~nm}$. O teste 
foi realizado em triplicata. A capacidade antioxidante das mostras foi expressa em Atividade Antioxidante Relativa (AAR) em relação aos padrões, de acordo com a Equação 3:

$A R R \%=\frac{A b s_{\text {amostra }} \times A b s_{\text {branco }}}{A b s_{\text {padrão }} x A b s_{\text {branco }}}$

\subsection{Atividade antioxidante (DPPH)}

As atividades antioxidantes dos dois extratos vegetais, jurema preta e cajueiro, foram determinadas a partir da adaptação da metodologia de Brand-Williams [14]. Inicialmente preparou-se a solução dos extratos vegetais na concentração de $1000 \mu \mathrm{g} . \mathrm{mL}^{-1}$. Posteriormente, pesou-se 2,5 mg de DPPH e adicionou-se a um balão volumétrico $(100 \mathrm{~mL})$ com uma solução previamente preparada de metanol $80 \%(\mathrm{v} / \mathrm{v})$ até aferir o menisco. Posteriormente foi inserido $3,9 \mathrm{~mL}$ da solução de DPPH em tubos de ensaios cobertos por papel alumínio e $0,1 \mathrm{~mL}$ da solução do extrato vegetal. $\mathrm{O}$ sistema foi mantido em repouso por 30 minutos ao abrigo da luz. Efetuou-se as leituras em um espectrofotômetro UV/VIS no comprimento de onda de $515 \mathrm{~nm}$. Os ensaios foram realizados em triplicatas e para o controle foi adicionado $0,1 \mathrm{~mL}$ da solução de metanol $80 \%$ (v/v) substituindo os extratos vegetais. A porcentagem de inibição foi calculada através dos valores de absorbâncias (Abs) utilizando a equação 4.

$$
\% \text { de inibição }=\frac{A b s_{\text {controle }}-A b s_{\text {amostra }}}{A b s_{\text {controle }}}
$$

\subsection{Avaliação da atividade antimicrobiana dos extratos vegetais}

Antes de avaliar a atividade antimicrobiana nos extratos vegetais, realizou-se uma secagem dos extratos na temperatura de $40^{\circ} \mathrm{C}$ utilizando uma estufa com circulação de ar durante um período de 24 horas. Para a avaliação antimicrobiana dos extratos de jurema preta e cajueiro, usando o teste de difusão em disco, submetidos aos micro-organismos da espécie Staphylococcus aureus. Para o ensaio de difusão em meio sólido, utilizou-se placas de Petri com o meio ágar nutritivo inoculado com suspensão bacteriana $\left(10^{-5}\right.$ na escala MacFarland). Com a ajuda de uma pipeta esterilizada, foram confeccionados pequenos poços no meio nutritivo e preenchidos com os extratos vegetais. Posteriormente, a placa foi incubada durante 48 horas na temperatura de $30^{\circ} \mathrm{C}$. Após o período de incubação, foi realizada a medida do halo de inibição com um paquímetro. Todas as análises foram realizadas em duplicatas.

\subsubsection{Avaliação da concentração mínima inibitória}

Para a análise da concentração mínima inibitória, utilizando a metodologia da NCCLS [15], foi preparada uma solução do extrato vegetal com concentração inicial de 0,5454 g.mL $\mathrm{mL}^{-1}$. Para o teste, foram utilizados 30 tubos de ensaio, em 10 sequências de três tubos, contendo $2 \mathrm{~mL}$ do meio caldo tripticaseína de soja (TBS). $\mathrm{Na}$ série zero, foram adicionados $2 \mathrm{~mL}$ da solução do extrato. De cada tubo da primeira série foram retirados $2 \mathrm{~mL}$ e transferidos para os tubos da próxima série. Repetiu-se esse procedimento até se obter um total de 9 diluições. Posteriormente, foram inoculados $0,5 \mathrm{~mL}$ de uma suspensão do micro-organismo Staphylococcus aureus (0,5 na escala MacFarland). Depois estes foram incubados em estufa a $30^{\circ} \mathrm{C}$ por um período de 24 horas. As concentrações dos biocidas utilizadas em cada série de diluição estão descritas na Tabela 1.

Tabela 1: Séries de diluição da concentração dos biocidas utilizados no teste CMI.

\begin{tabular}{cc}
\hline SÉRIE DE DILUIÇÃO & CONCENTRAÇÃO (G/ML) \\
\hline 0 & 0,2727 \\
1 & 0,1364 \\
2 & 0,0682 \\
3 & 0,0341 \\
4 & 0,0170 \\
5 & 0,0085 \\
6 & 0,0043 \\
7 & 0,0021 \\
8 & 0,0011 \\
9 & 0,0005 \\
\hline
\end{tabular}


As confirmações dos testes de CMI em tubos de ensaio foram realizadas através de um plaqueamento, com placas de Petri contendo meio ágar nutriente. Como as soluções do extrato nos tubos com o meio TBS eram turvas e não davam para identificar o crescimento do micro-organismo, todas as amostras foram repicadas para as placas, e incubadas durante $48 \mathrm{~h}$ a $30^{\circ} \mathrm{C}$.

\subsection{Preparação dos filmes}

Os filmes de PBAT com o extrato vegetal foram produzidos em duplicata nas concentrações 1,5 e $10 \%$ $(\mathrm{m} / \mathrm{m})$. O polímero foi dissolvido em $20 \mathrm{~mL}$ de clorofórmio, na presença de agitação, e os extratos secos foram dissolvidos em $5 \mathrm{~mL}$ de etanol cada. Então, as soluções foram misturadas e agitadas durante 15 minutos. Após isso, foram postas em placas de Petri e colocadas em repouso para evaporação do solvente.

\subsection{Cinética de migração}

A metodologia deste ensaio foi adaptada de CARVALHO et al. [16]. Para isso, foi cortada uma amostra de 2,5 x 2,5 $\mathrm{cm}^{2}$ do filme de PBAT incorporado com extrato de cajueiro e ela foi dobrada em formato de "sanfona", para que, durante o teste, todas as partes do corpo de prova estivessem em contato com o meio. Em seguida, a amostra foi colocada em um tubo de ensaio e $10 \mathrm{~mL}$ de meio simulante alimentício etanólico $(10 \% \mathrm{v} / \mathrm{v})$ foram adicionados. Os tubos foram tampados e cobertos com papel alumínio para evitar a exposição à luz e mantidos em constante agitação a $25^{\circ} \mathrm{C}$.

Após 30, 60, 90, 180, 250 e 1440 minutos, até a estabilização da migração, foram realizadas as leituras das absorbâncias em espectrofotômetro UV/VIS no comprimento de onda de $450 \mathrm{~nm}$, determinado após uma varredura na região do visível usando a solução etanólica de extrato de $1000 \mu \mathrm{g} \cdot \mathrm{mL}^{-1}$ e etanol $(10 \%$ v/v) como o branco. A curva de calibração foi feita utilizando soluções do extrato, com diferentes concentrações.

Uma equação de cinética de primeira ordem (Equação 5) foi usada no ajuste dos dados da migração do extrato de cajueiro do filme de PBAT em função do tempo de contato com o etanol

Os resultados de concentração foram expressos em $\mu \mathrm{g} \cdot \mathrm{mL}^{-1}$.

$C_{(t)}=C_{e q}+A \cdot e^{(-k \cdot t)}$

$\mathrm{Na}$ qual $\mathrm{C}_{(\mathrm{t})}$ é a concentração medida em função do tempo em $\mu \mathrm{g} \cdot \mathrm{mL}^{-1}, \mathrm{C}_{\mathrm{eq}}$ é o valor da concentração de equilíbrio no estado estacionário em $\mu \mathrm{g} \cdot \mathrm{mL}^{-1}$, $\mathrm{t}$ é o tempo em minutos, $\mathrm{k}$ é a taxa de migração $\left(\mu \mathrm{g} \cdot \mathrm{mL}^{-}\right.$ $\left.{ }^{1} \cdot \mathrm{min}^{-1}\right)$ e A é um parâmetro de ajuste.

\subsection{Análise estatística}

Os dados dos rendimentos de extração, capacidade antioxidante total e atividade antioxidante (DPPH) foram analisados por análise de variância (ANOVA), utilizando o software Statistica, versão 10.0.228.8. O teste de Duncan foi utilizado para determinar diferenças em um nível de significância de $5 \%(p \leq 0,05)$.

\section{RESULTADOS}

\subsection{Avaliações dos rendimentos dos extratos vegetais}

Observando a Tabela 2 pode-se notar os percentuais de rendimento para os extratos vegetais de jurema preta e cajueiro.

Tabela 2: Rendimento da extração da casca de cajueiro e jurema preta.

\begin{tabular}{c|c|c}
\cline { 2 - 3 } \multicolumn{2}{c|}{ JUREMA PRETA } & CAJUEIRO \\
\hline TEMPO DE EXTRAÇÃO (H) & RENDIMENTO (\%) & RENDIMENTO (\%) \\
\hline 4 & $11,60^{\mathrm{a}} \pm 1,39$ & $22,90^{\mathrm{a}} \pm 0,68$ \\
6 & $15,30^{\mathrm{b}} \pm 0,46$ & $24,00^{\mathrm{a}} \pm 0,28$ \\
8 & $15,50^{\mathrm{b}} \pm 0,90$ & $23,10^{\mathrm{a}} \pm 1,13$ \\
\hline
\end{tabular}

*Médias seguidas de letras iguais na vertical não diferem significativamente ( $p>0,05)$ pelo teste de Duncan.

A partir do teste estatístico Duncan para o extrato de jurema preta (Tabela 2), percebe-se que para o tempo de 4 horas foi obtido um menor percentual $(11,60 \%)$ de rendimento, já para os tempos de 6 e 8 horas 
não foi constatado diferença significativa entre os tempos de extração. Portanto, foi adotado o tempo de seis horas como o tempo escolhido para a extração dessa espécie. Isso ocorre porque a medida que o tempo de permanência a temperatura aumenta, pode-se acarretar na degradação dos compostos orgânicos responsáveis pelas suas propriedades. Para o extrato vegetal de cajueiro nos resultados estatísticos foram constatados que não houve diferença significativa entre os tempos de extração de 4,6 e 8 horas, consequentemente, foi escolhido o tempo de 4 horas para a sua extração.

\subsection{Teste da presença de flavonoides}

Na Tabela 3 estão apresentados os valores medidos da concentração de flavonoides encontradas nas amostras dos extratos de cajueiro e jurema preta.

Tabela 3: Concentração de flavonoides nos extratos.

\begin{tabular}{ccc}
\hline Amostra & Concentração $\left(\boldsymbol{\mu g} \cdot \mathbf{m L}^{-1}\right)$ & $\mathbf{m g E Q U} / \mathbf{g}$ \\
\hline jurema preta & $5,4989^{\mathrm{a}} \pm 0,4406$ & 5,49 \\
cajueiro & $1,1140^{\mathrm{b}} \pm 0,1089$ & 1,14 \\
\hline
\end{tabular}

*Médias seguidas de letras iguais na vertical não diferem significativamente $(\mathrm{p}>0,05)$ pelo teste de Duncan

Nota-se que o extrato de jurema preta $\left(5,4989 \mu \mathrm{g} \cdot \mathrm{ml}^{-1}\right)$ apresenta uma maior concentração de flavonoides em relação ao cajueiro $\left(1,1140 \mu \mathrm{g} \cdot \mathrm{ml}^{-1}\right)$. Este comportamento pode ser um possível indicativo de uma maior atividade antioxidante, uma vez que esse grupo pode atuar como antioxidante natural devido à sua capacidade de estabilizar radicais livres e espécies reativas de oxigênio por causa dos grupos hidroxilas ligados à estrutura do anel [17].

\subsection{Teste da presença de fenólicos totais}

O método de Folin-Ciocalteau é o mais usado para determinação dos fenóis totais, tem como princípios a utilização de um reagente que é formado pela mistura dos ácidos fosfomolibídico e fosfotunguístico, onde o molibdênio (Mo) e o tungstênio (W) encontram-se no estado de oxidação 6+, e esse reagente oxida os fenolatos, reduzindo os ácidos a um complexo azul Mo-W. Na Tabela 4 a seguir estão apresentadas as concentrações de fenólicos totais nos extratos de jurema preta e cajueiro.

Tabela 4: Concentração de fenólicos totais nos extratos.

\begin{tabular}{ccc}
\hline Amostra & Concentração $\left(\boldsymbol{\mu g} \cdot \mathbf{m L}^{-1}\right)$ & $\mathbf{m g E G A} / \mathbf{g}$ \\
\hline cajueiro & $87,268^{\mathrm{a}} \pm 6,250$ & 436,34 \\
jurema preta & $50,584^{\mathrm{b}} \pm 0,759$ & 272,92 \\
\hline
\end{tabular}

*Médias seguidas de letras iguais na vertical não diferem significativamente ( $p>0,05)$ pelo teste de Duncan.

Diante dos resultados, foi observado que a concentração de fenólicos totais no extrato de cajueiro $\left(87,268 \mu \mathrm{g} \cdot \mathrm{mL}^{-1}\right)$ foi maior que no de jurema preta $\left(50,584 \mu \mathrm{g} \cdot \mathrm{mL}^{-1}\right)$, variando significativamente, apesar do segundo ter apresentado uma concentração de flavonoides maior. Este fato pode estar relacionado a presença de outros grupos fenólicos, como por exemplo, os taninos que podem provocar uma maior ação antioxidante. Os taninos podem atuar interrompendo a cadeia de reação radicalar ao reagir com radicais e formarem produtos mais estáveis que o radical livre precursor, termodinamicamente ou pela formação de complexos. Os fenólicos são classificados como antioxidantes primários [18].

$\mathrm{Na}$ literatura foi encontrado os teores de fenólicos totais para os extratos de cajueiro e jurema preta que, respectivamente, apresentaram os valores de 345,16 e 678,00 mgEAG/g (relativo ao padrão de ácido gálico por gramas de extrato) [19, 20]. Comparando com valores observados na literatura, o extrato vegetal de cajueiro apresentou um maior teor de fenólicos, enquanto que o extrato de jurema preta apresentou um valor menor.

Essas variações da composição dos extratos vegetais ocorrem devido aos metabolitos secundários, uma vez que os princípios ativos da planta são produzidos a partir de um mecanismo de defesa do vegetal, ocorrendo quando se encontra em condições de estresse fisiológico (falta de nutrientes e água), fatores ambientais (clima) e proteção contra predadores ou micro-organismos patógenos [21].

\subsection{Capacidade antioxidante total dos extratos vegetais}


A capacidade antioxidante das amostras, apresentada na Tabela 5, foi expressa em atividade antioxidante Relativa (AAR), de acordo com a Equação 1.

Tabela 5: Capacidade antioxidante dos extratos vegetais de cajueiro e jurema preta.

\begin{tabular}{cc}
\hline AMOSTRA & CAPACIDADE ANTIOXIDANTE (\%) \\
\hline cajueiro & $43,91^{\mathrm{a}} \pm 3,18$ \\
jurema preta & $41,77^{\mathrm{a}} \pm 6,30$ \\
Padrão & 100 \\
Branco & 0 \\
\hline
\end{tabular}

*Médias seguidas de letras iguais na vertical não diferem significativamente ( $>>0,05)$ pelo teste de Duncan.

Logo, dos dados analisados, foi possível verificar que não houve variação significativa na capacidade antioxidante de ambas as amostras. Tanto o extrato de cajueiro quanto o de jurema preta obtiveram atividades significativas em relação ao ácido ascórbico, utilizado como padrão, assim como ARANDA-SOUZA et al., [22] também observaram em outra planta encontrada no sertão nordestino, a parkinsonia aculeata L. Na literatura não foi encontrado nenhum estudo da capacidade antioxidante total pelo método de redução do fosfomolibdênio para os extratos de cajueiro e jurema preta.

\subsection{Atividade Antioxidante dos extratos vegetais (DPPH)}

A atividade antioxidante das amostras de extrato vegetal de cajueiro e jurema preta pelo método do DPPH está apresentada na Tabela 6.

Tabela 6: Atividade antioxidante dos extratos vegetais pelo método de captura do radical livre do DPPH.

\begin{tabular}{cc}
\hline AMOSTRA & ATIVIDADE ANTIOXIDANTE (\%) \\
\hline cajueiro & $87,71^{\mathrm{a}} \pm 0,45$ \\
jurema preta & $86,08^{\mathrm{b}} \pm 0,13$ \\
\hline
\end{tabular}

*Médias seguidas de letras iguais na vertical não diferem significativamente ( $>0,05)$ pelo teste de Duncan.

Observou-se que a atividade antioxidante do extrato de cajueiro, 87,71\%, foi estatisticamente significativa quando relacionada com o extrato de jurema preta, 86,08\%, e ambos apresentaram valores significativos de sequestro de radicais livres. CHAVES et al., [23] encontraram percentuais semelhantes de sequestro de DPPH para extratos da casca de cajueiro, provavelmente, oriundos da presença de ácidos anacárdicos e taninos, e FRANCO et al., [24] também observaram valores semelhantes para jurema preta devido à presença de compostos fenólicos.

\subsection{Avaliação da atividade antimicrobiana dos extratos vegetais}

A média dos halos de inibição obtida pelos extratos de jurema preta e cajueiro no teste de difusão em disco está indicada na Tabela 7 e Figura 1.

Tabela 7: Média dos halos de inibição em 48 horas de incubação.

\begin{tabular}{cc}
\hline AMOSTRAS & RAIO (MM) \\
\hline cajueiro & 16,05 \\
jurema preta & 9,85 \\
\hline
\end{tabular}

No teste de difusão em disco, o extrato de jurema preta apresentou inibição contra Staphylococcus aureus, com halo de 9,85 mm de raio, Tabela 7 e Figura 1, o que corroborou com os resultados apresentados por PEREIRA [25], que observou a formação de halos de inibição entre 5-20 mm ao se variar concentrações de extrato contra este micro-organismo. 


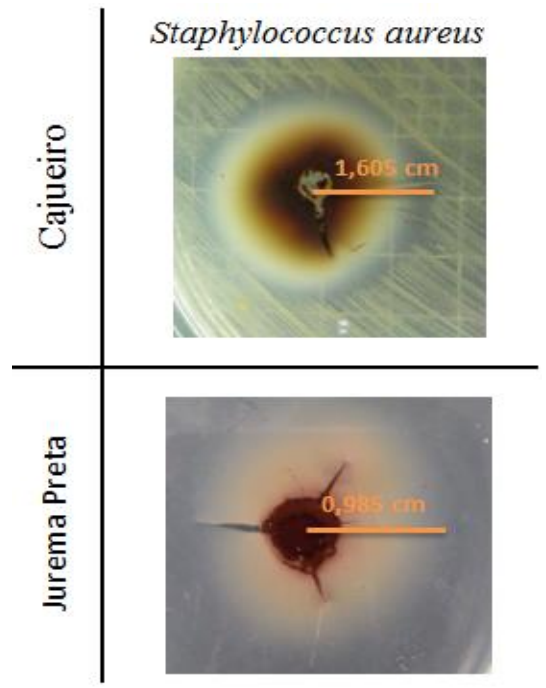

Figura 1: Teste de atividade antimicrobiana dos extratos vegetais.

Como os halos de inibição do cajueiro foram maiores que o da jurema preta, indicando uma atividade mais efetiva, foi avaliada a CMI desse extrato, como também foi usado como aditivo para os filmes de PBAT.

\subsubsection{Avaliação da concentração mínima inibitória}

A Tabela 8 mostra os resultados da ação do extrato de cajueiro em diferentes concentrações contra a ação do Staphylococcus aureus.

Tabela 8: Avaliação da Concentração Mínima Inibitória (CMI) no plaqueamento, com extrato de cajueiro em diferentes concentrações, contra Staphylococcus aureus.

\begin{tabular}{ccc}
\hline SÉRIE DE DILUIÇÃO & $\begin{array}{c}\text { CONCENTRAÇÃO } \\
\left(\mathbf{g} \cdot \mathbf{m L}^{-1}\right)\end{array}$ & $\begin{array}{c}\text { CRESCIMENTO DO } \\
\text { MICRO-ORGANISMO }\end{array}$ \\
\hline 0 & 0,2727 & - \\
1 & 0,1364 & - \\
2 & 0,0682 & - \\
3 & 0,0341 & - \\
4 & 0,0170 & + \\
5 & 0,0085 & + \\
6 & 0,0043 & + \\
7 & 0,0021 & + \\
8 & 0,0011 & + \\
9 & 0,0005 & \\
\hline
\end{tabular}

$\mathrm{Na}$ avaliação da CMI do extrato de cajueiro, pode ser observado que, a partir de $0,0085 \mathrm{~g} \cdot \mathrm{mL}^{-1}$, o extrato não apresentou ação biocida e, como pode ser notado na Figura 2, a provável CMI é correspondente a diluição $4\left(0,0170 \mathrm{~g} \cdot \mathrm{mL}^{-1}\right)$. Contudo, a observação visual foi complexa devido ao extrato apresentar fibras, que podem ser confundidas com o micro-organismo, e coloração muito escura, o que influencia na turbidez do meio. 


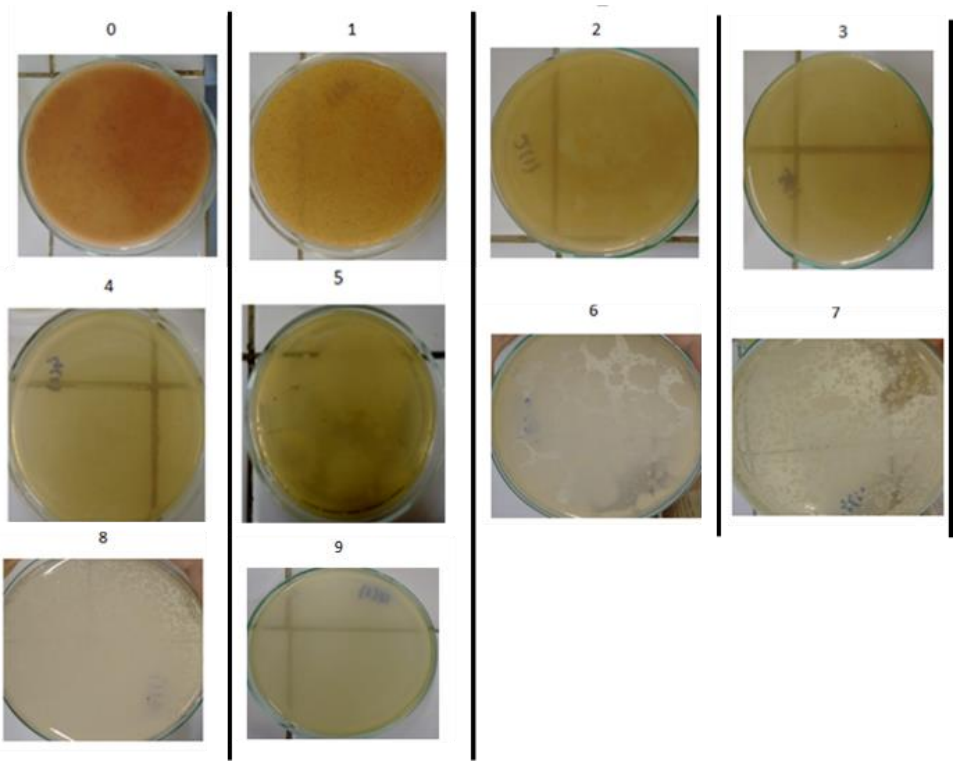

Figura 2: Plaqueamento com extrato de Cajueiro contra Staphylococcus aureus nas concentrações correspondente as series de diluição 0 a 9 .

SILVA et al., [28] observaram ação antimicrobiana do extrato hidroalcoólico da casca do caule do cajueiro frente a amostras de Staphylococcus aureus resistentes e sensíveis à meticilina, obtidas a partir de pacientes internados do Hospital Universitário/Universidade Federal da Paraíba. Além disso, SANTOS e ALVES [29] relataram que essa planta é uma das mais citadas com atividade antimicrobiana relevante sobre o Staphylococcus aureus, sendo sua ação relacionada à presença de taninos [30].

GONÇALVES et al., [26] também observaram a ação inibitória M. tenuiflora sobre Streptococcus pyogenes, Proteus mirabilis, Shigella sonnei, Staphylococcus aureus e S. spp. coagulase-negativa. Essa atividade antimicrobiana pode estar relacionada à presença de taninos e flavonoides [6], uma vez que BEZERRA [27] avaliou a fitoquímica desse extrato e a presença dos compostos: taninos, flavonas, catequinas leucoantocianinas, saponina, triterpenóides e alcaloides.

\subsection{Aspectos físicos dos filmes de PBAT incorporado com extrato de cajueiro}

Os filmes obtidos podem ser observados na Figura 3, na qual pode-se observar que os correspondentes às concentrações de 5 e $10 \%$ de extrato de cajueiro, não aderiram bem ao polímero. Isso pode ter ocorrido devido à diferença de polaridade entre o solvente, clorofórmio, e o extrato etanólico, resultando em uma separação. Desta forma, foram utilizados os filmes com $1 \%$ de extrato no ensaio de migração.
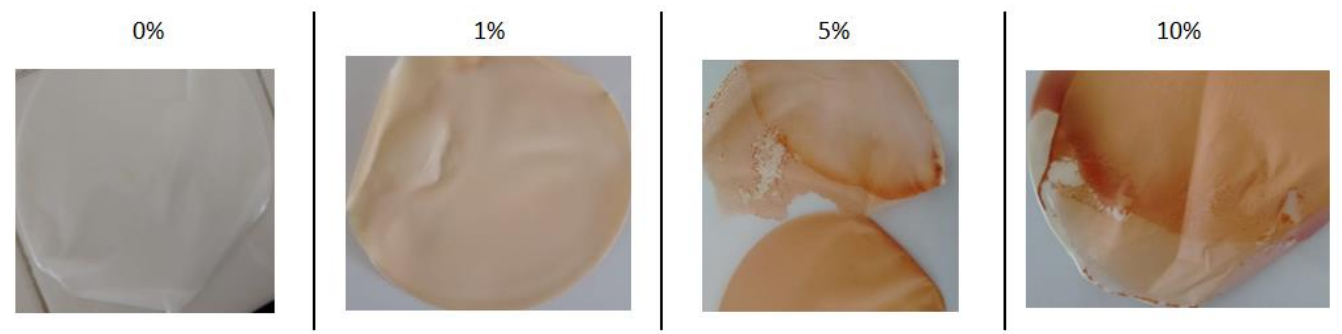

Figura 3: Filmes de PBAT incorporados com 0, 1, 5 e $10 \%$ de extrato de cajueiro.

Para a determinação do parâmetro k, taxa de migração, foi utilizada a representação de gráfica de $\ln (\mathrm{C})$ versus $\mathrm{t}$, correspondente à parte linear da curva experimental, cuja inclinação 0,0167 é a constante. Então $0,0167 \mu \mathrm{g} \cdot \mathrm{mL}^{-1} \cdot \mathrm{min}^{-1}$ é a velocidade com a qual o extrato migra da matriz polimérica para o meio simulante.

A concentração de equilíbrio adotada foi a concentração correspondente a 250 minutos, $43,405 \mu \mathrm{g} . \mathrm{mL}^{-1}$, onde já é possível observar, na Figura 4, o início da estabilização da concentração do extrato no meio etanólico e a taxa de migração já é bem reduzida.

Para a determinação do parâmetro de ajuste, foi utilizado o ponto experimental correspondente a 180 minutos e o valor encontrado foi -33,24. A partir disso, foi definido um modelo cinético para a migração do extrato de cajueiro do filme de PBAT para o meio etanólico, representado pela Equação 6. 
$C_{(t)}=43,405-33,24 \cdot e^{(-0,0167 . t)}$

Com a equação, é possível traçar a curva teórica da migração, como mostrado na Figura 4, juntamente com a curva experimental.

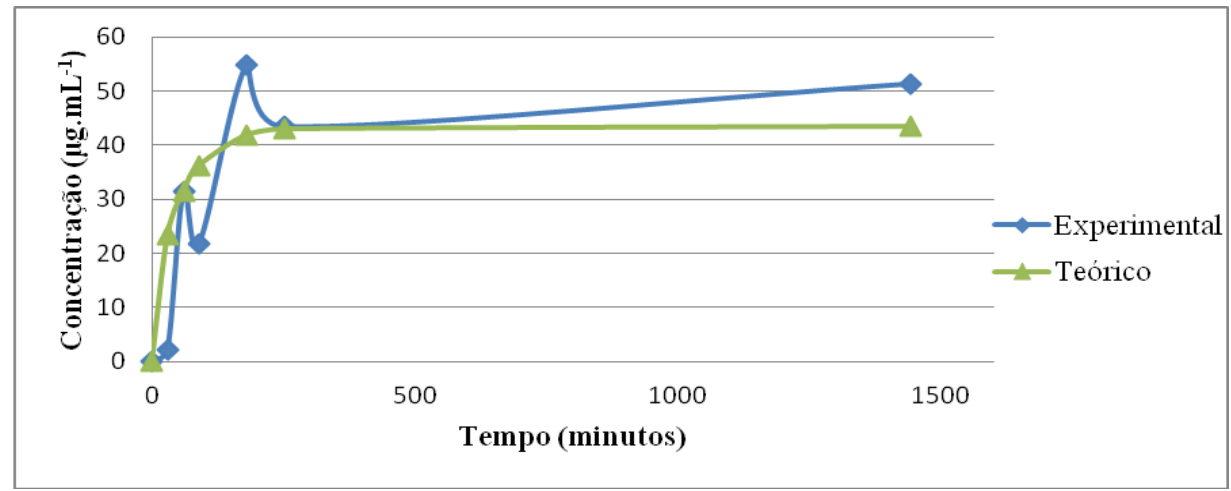

Figura 4: Gráfico das curvas experimental e teórica de concentração versus tempo.

\section{CONCLUSÕES}

Foram avaliadas as potencialidades dos extratos vegetais de jurema preta (Mimosa tenuiflora) e cajueiro (Anacardium occidentale Linn), a fim de aplica-los como aditivos em embalagens biodegradáveis ativas antioxidantes e antimicrobianas de PBAT. Os tempos escolhidos de extração para os extratos de jurema preta e cajueiro foram 6 e 4 horas, respectivamente. Foram realizados testes acerca da presença dos grupos químicos flavonoides e fenólicos totais, por apresentarem relações com a atividade antioxidante e, apesar do extrato de jurema preta ter apresentado um teor de flavonoides maior que o de cajueiro, este obteve uma maior concentração de fenólicos totais, indicando a presença de outros fenólicos além dos flavonoides, como os taninos. Suas capacidades antioxidantes evidenciaram atividades significativas em relação ao padrão ácido ascórbico, pelo método do fosfomolibdênio, e os percentuais pelo método do sequestro de DPPH foram maiores para o extrato de cajueiro e ambos apresentaram valores significativos. A atividade antimicrobiana dos extratos contra Staphylococcus aureus foi comprovada, na qual o de cajueiro apresentou halos maiores de inibição que o de jurema preta e, por isso, foi escolhido para determinar sua concentração mínima inibitória, 0,0170 g. $\mathrm{mL}^{-1}$, e ser incorporado ao polímero. Quanto maior a concentração do extrato em filmes PBAT, menor a aderência e homogeneização do extrato, provavelmente devido à polaridade e solvente utilizado para a produção do filme polimérico. $\mathrm{O}$ filme aditivado com $1 \%$ de extrato vegetal cajueiro foi escolhido para o teste de migração no meio simulante etanólico, por meio do qual foi possível determinar um modelo cinético de migração em meio simulante alimentício.

\section{AGRADECIMENTOS}

A Universidade Federal de Pernambuco, ao Laboratório de Petroquímica, ao Laboratório de Microbiologia, Laboratório de Bioprocessos e ao Grupo de Instrumentação e análises químicas (GIAQ) (UAST/UFRPE) pelo apoio institucional. A Fundação de Amparo à Ciência e Tecnologia de Pernambuco (FACEPE) e ao Conselho Nacional de Desenvolvimento Científico e Tecnológico (CNPQ) pelas bolsas concedidas.

\section{BIBLIOGRAFIA}

[1] SOUZA, A.C., BENZE, R., FERRÃO, et al., "Cassava starch biodegradable films: Influence of glycerol and clay nanoparticles content on tensile and barrier properties and glass transition temperature", LWT - Food Science and Technology, v. 46, n. 1, pp. 110-117, 2012.

[2] MORAES, A.R.F., GOUVEIA, L.E.R., SOARES, N.F.F., et al., "Desenvolvimento e avaliação de filme antimicrobiano na conservação de manteiga", Rev. Ceres, v. 57, n.3, pp. 283-291, mai/jun, 2007.

[3] FANG, Z., ZHAO, Y., WARNER, R.D., et al., "Active and Intelligent Packaging in Meat Industry." Trends in Food Science \& Technology, v. 61, n. 2, p. 60-71, 2017.

[4] SOARES, N.D.F.F., SILVA, W.A., PIRES, A.C.S., et al., "Novos desenvolvimentos e aplicações em embalagens de alimentos", Ceres, v. 56, n. 4, pp. 370-378, 2009.

[5] PIRES, M., SANTOS, R.V., CHIES, A.P., et al., "Efeito da migração de composto antimicrobiano nas 
propriedades finais de selagem de filme poliolefínico", Polímeros, v. 24, pp. 237-242, 2014.

[6] LOZOYA, X., NAVARRO, V., ARNASON, J.T., et al., "Experimental evaluation of Mimosa tenuiflora (Wild.) Poir. (Tepeschohuite) I. Screening of the antimocrobial properties of bark extracts", Archivos de Investigación Medica, v.20, n.1, pp. 87-93, 1989.

[7] LAURENS, A., MBOUP, S., GIONO-BARBER, P., et al., "Etude de l'action antibacterenne d'extraits d'Anacardium occidentale L.", Annales Pharmaceutiques Françaises, v.40, pp. 143-146, 1992.

[8] ZEHETMEYER, G., MEIRA, S.M.M., SCHEIBEL, J.M., et al.," Influence of melt processing on biodegradable nisin-PBAT films intended for active food packaging applications", J. Appl. Polym. Sci., v. 133, 2016.

[9] TAVARES, L.B., ROSA, D.S., "Stabilization effect of kraft lignin into PBAT:Thermal analyses approach." Rev. Mat. v.24, n.3, 2019.

[10] SOUSA, J.C., ARRUDA, S.A., LIMA, J.C., et al., "Crystallization kinetics of poly (butylene adipate terephthalate) in biocomposite with coconut fiber." Revista Matéria, v.24, n.3, 2019.

[11] OLIVEIRA, V.B., ZUCHETTO, M., OLIVEIRA, C.F., et al., "Efeito de diferentes técnicas extrativas no rendimento, atividade antioxidante, doseamentos totais e no perfil por clae-dad de dicksonia sellowiana (presl.). Hook, dicksoniaceae.”, Rev. Bras. Pl. Med., v.18, n.1, pp.230-239, 2016.

[12] FOLIN, O., CIOCALTEU, V., "On tyrosine and tryptophane determinations in proteins", The Journal of Biological Chemistry, v. 73, pp. 627-650, 1927.

[13] NIBIR Y.M., SUMIT, A.F., AKHAND, A.A., et al., "Comparative assessment of total polyphenols, antioxidant and antimicrobial activity of different tea varieties of Bangladesh", Asian Pacific Journal of Tropical Biomedicine, v. 7, n. 4, pp. 352-357, 2017.

[14] BRAND-WILLIAMS, W., CUVELIER, M.E., BERSET, C., "Use of a free radical method to evaluate antioxidant activity", LWT - Food Science and Technology, v. 28, n. 1, pp. 25-30, 1995.

[15] NCCLS. "Methods for Dilution Antimicrobial Susceptibility Tests for Bacteria That Grow Aerobically; Approved Standard-Sixth Edition", NCCLS document M7-A6 (ISBN 1-56238-486-4), Pennsylvania, 2003.

[16] CARVALHO, D.M., TAKEUCH, K.P., GERALDINE, R.M., et al., "Active film of cellulose acetate incorporated with nanosuspension of curcumin”, Polimeros, v. 27, n. especial, pp. 70-76, 2017.

[17] BEHLING, E.B., SENDÃO, M.C., FRANCESCATO, H.D.C., et al., "Flavonóide quercetina: aspectos gerais e ações biológicas", Alim. Nutr., v. 15, n. 3, pp. 285-292, 2004.

[18] ANGELO, P.M., JORGE, N., "Compostos fenólicos em alimentos - uma breve revisão", Revista Instituto Adolfo Lutz, São Paulo, v. 66, pp. 232-240, 2007.

[19] PEREIRA, A.P.C. CARACTERIZAÇÃO QUÍMICA E POTENCIAL BIOLÓGICO DE METABOLITOS SECUNDÁRIOS DE Commiphora leptophloeos (Mart.) J.B.Gillett. 2016. 106 f. Tese (Doutorado) - Curso de Ciências Biológicas, Centro de Ciências Biológicas, Universidade Federal de Pernambuco, Recife, 2016.

[20] CRUZ, M.P. ISOLAMENTO E IDENTIFICAÇÃO DE COMPOSTOS BIOATIVOS DE Mimosa hostilis BENTH. 2013. 204 f. Tese (Doutorado) - Curso de Química Orgânica, Instituto de QuÍmica, Universidade Federal da Bahia, Salvador, 2013.

[21] OETTING, L.L. Extratos vegetais como promotores do crescimento de leitões recém-desmamados. 2005. 80 f. Tese (Doutorado) - Curso de Ciência Animal e Pastagens, Universidade de São Paulo, São Paulo, 2005.

[22] ARANDA-SOUZA, M.A., SOUZA, R.M., JÚNIOR, C.A.S., et al., "Antioxidant potential and total phenolic content of leaf extracts from Parkinsonia aculeata L. cultivated in Brazilian Caatinga biome", Current Topics in Phytochemistry, v. 12, pp. 95-101, 2012.

[23] CHAVES, M.H., CITÓ, A.M.G.L., LOPES, J.A.D., et al., "Fenóis totais, atividade antioxidante e constituintes químicos de extratos de Anacardium occidentale L., Anacardiaceae", Revista Brasileira de Farmacognosia, v. 20, n. 1, pp. 106-112, 2010.

[24] FRANCO, S.P.B., COSTA, J.G., PAVÃO, J.M.S.J., "Análise cromatográfica e avaliação do potencial antioxidante da mimosa tenuiflora", In: CONADIS, Dez. 2018.

[25] PEREIRA, A.V., "Estudo da ação dos extratos de Mimosa tenuiflora (Wild) Poiret e Piptadenia stipulacea (Benth) Ducke sobre cepas microbianas de mastite em búfalas", Dissertação de M.Sc., Centro de Saúde e Tecnologia Rural, Universidade Federal de Campina Grande, Campina Grande, PB, Brasil, 2010.

[26] GONÇALVES, A.L., ALVES FILHO, A., MENEZES, H., "Efeitos antimicrobianos de algumas árvores medicinais nativas nas conjuntivites infecciosas", O Biológico, v. 68, 2006.

[27] BEZERRA, D.A.C., "Estudo Fitoquímico, Bromatológico e Microbiológico de Mimosa tenuiflora (Wild) Poiret e Piptadenia stipulacea (Benth) Ducke", Dissertação de M.Sc., Universidade Federal de Campina Grande, Campina Grande, PB, Brasil, 2008.

[28] SILVA. J.G., SOUZA, I.A., HIGINO, J.S., et al., "Atividade antimicrobiana do extrato de Anacardium 
occidentale Linn. em amostras multiresistentes de Staphylococcus aureus", Revista Brasileira de Farmacognosia, pp. 572-577, Out./Dez, 2007.

[29] SANTOS S.J.D., ALVES F., "Análise comparativa da ação de extratos de plantas com atividade antimicrobiana (in vitro) sobre cepas de Staphylococcus aureus", Periódico científico do núcleo de Biociências, v.2, n.04, pp. 12-19, 2012.

[30] MOTA, R.A., MEDEIROS, E.S., SANTOS, M.V., et al., "Participação dos Staphylococcus spp na etiologia das mastites e bovinos leiteiros no estado de Pernambuco (Brasil)", Revista Ciência Animal Brasileira, v.13, n.1, pp. 124-130, jan./mar. 2012.

ORCID

Ivo Diego de Lima Silva Flávia Suellen Melo de Oliveira Michelle Felix de Andrade Andréa Monteiro Santana Silva Brito Fernando Hallwass Glória Maria Vinhas https://orcid.org/0000-0001-7441-2305

https://orcid.org/0000-0002-4502-2735

https://orcid.org/0000-0002-4798-1403

https://orcid.org/0000-0002-2026-130X

https://orcid.org/0000-0002-8304-1427

https://orcid.org/0000-0001-5073-609X 